\title{
Localizing Neuronal Somata from Multi-Electrode Array In-Vivo Recordings using Deep Learning
}

\author{
Alessio P. Buccino*, Torbjørn V. Ness ${ }^{\dagger}$, Gaute T. Einevoll ${ }^{\dagger \ddagger}$, Gert Cauwenberghs ${ }^{\S}$, and Philipp D. Häfliger*. \\ *Department of Informatics, University of Oslo, Oslo, Norway \\ ${ }^{\dagger}$ Faculty of Science and Technology, Norwegian University of Life Sciences, Ås, Norway \\ ${ }^{\ddagger}$ Department of Physics, University of Oslo, Oslo, Norway \\ $\S$ Department of Bioengineering, University of California San Diego, La Jolla, CA, USA \\ Email: alessiob@ifi.uio.no, torbjorn.ness@nmbu.no, gaute.einevoll@nmbu.no, gert@ucsd.edu, hafliger@ifi.uio.no
}

\begin{abstract}
With the latest development in the design and fabrication of high-density Multi-Electrode Arrays (MEA) for in-vivo neural recordings, the spatiotemporal information in the recorded signals allows for refined estimation of a neuron's location around the probe. In parallel, advances in computational models for neural activity enables simulation of recordings from neurons with detailed morphology. Our approach uses deep learning algorithms on a large set of such simulation data to extract the 3D position of the neuronal somata. Multicompartment models from 13 different neural morphologies in layer 5 (L5) of the rat's neocortex are placed at random locations and with different alignments with respect to the MEA. The sodium trough and repolarisation peak images on the MEA serve as input features for a Convolutional Neural Network $(\mathrm{CNN})$, which predicts the neural location robustly and with low error rates. The forward modeling/machine learning approach yields very accurate results for the different morphologies and is able to cope with different neuron alignments.
\end{abstract}

\section{INTRODUCTION}

Neurons are spatially arranged throughout the entire brain and their spatial organization is an important aspect of neural circuits. Therefore, we need estimates or triangulation of the relative position between the recorded and sorted neurons and a probe, such as a Multi-Electrode Array (MEA). In spite of the strict power and size constraint for in-vivo silicon probes, new prototypes with high-density contacts are being developed and tested in-vivo. In Schröder et al. [1], a MEA with 256 recording sites (a $16 \times 16$ array) was used to monitor the functional electrical imaging [2] of event-related Local Field Potentials (LFPs) in the rat's barrel cortex. Such high spatial information density can be exploited to refine localization techniques.

Nevertheless, neural localization from extracellular recordings is a difficult and ill-posed problem; ideally, one would want to estimate the current source density (CSD) of each voxel in proximity of the probe. A voxel size small enough to obtain accurate results would require a very large number of recording sites and, moreover, multiple valid solutions are usually possible. Assuming simple neuronal models simplifies the problem and can make the solution unique. Examples of models used in previous studies are monopole and dipole current source models [3], as well as line source models [4]. More recently [5], modified ball-and-stick models [6] have represented pyramidal neurons (the ball is the soma, the stick is the apical dendrite) and the soma position has been predicted by minimizing the error between the spatial distribution of the potential on the MEA sites generated by the ball and stick model and the 'ground truth' spatial distribution, i.e. the one generated by detailed neural morphologies.

This latter approach shows improved results, but still has limitations: first, the ball-and-stick model only describes a pyramidal morphology; second, it only considers 3D shifts of the neurons with respect to the MEA, without respective alignments or rotations; and third, only a single time point, namely the sodium peak, is used as input to the optimization algorithm. In this paper, we attempt to address the abovementioned limitations and we try to do so by completely reversing the standard inverse approach: we use forward modeling [6] with detailed neural models [7] to build a simulated dataset before applying machine learning techniques to make inferences on the data. We leverage on the complexity and detail of the multi-compartment models in order to overcome some of the limitations of previous approaches. By including different cell types, the prediction is not tuned to a specific neural morphology. Moreover, we investigate the effect of having different alignments between the neuron and the MEA, and extend the feature set by including the repolarisation peak as well as the sodium peak.

Our computation is based on the 2D electrode distribution of the MEA prototype in [1]. A Convolutional Neural Network with a machine learning algorithm exploits the rich spatial information by this geometry for improved prediction.

The rest of the article is organized as follows: in section II we describe the methods used for cell and recording simulations and for the deep learning technique; in section III the results of the localization with various approaches are presented; and in section IV we contextualize and discuss this work by drawing its potentials and highlighting limitations and future directions.

\section{Methods}

\section{A. Cell types and morphologies}

For this study we first of all needed to generate simulated spike recordings from various neuronal types. The Blue Brain 


\begin{tabular}{ccccc} 
model name & layer & m-type & e-type & cell_id \\
\hline L5_BP_bAC217_1 & L5 & BP & bAC217 & 1 \\
L5_BTC_bAC217_1 & L5 & BTC & bAC217 & 1 \\
L5_ChC_cACint209_1 & L5 & ChC & cACint209 & 1 \\
L5_DBC_bAC217_1 & L5 & DBC & bAC217 & 1 \\
L5_LBC_bAC217_1 & L5 & LBC & bAC217 & 1 \\
L5_MC_bAC217_1 & L5 & MC & bAC217 & 1 \\
L5_NBC_bAC217_1 & L5 & NBC & bAC217 & 1 \\
L5_NGC_bNAC219_1 & L5 & NGC & bAC217 & 1 \\
L5_SBC_bNAC219_1 & L5 & SBC & bAC217 & 1 \\
L5_STPC_cADpyr232_1 & L5 & STPC & cADpyr232 & 1 \\
L5_TTPC1_cADpyr232_1 & L5 & TTPC1 & cADpyr232 & 1 \\
L5_TTPC2_cADpyr232_1 & L5 5 & TTPC2 & cADpyr232 & 1 \\
L5_UTPC_cADpyr232_1 & L5 & UTPC & cADpyr232 & 1 \\
& TABLE I & &
\end{tabular}

CELL TYPES USED IN SIMULATIONS: model name IS THE FULL MODEL NAME THAT CAN BE FOUND IN [8]

Project repository [7], [8] has been chosen and used as source for the detailed neuronal models. The models are from juvenile rat somatosensory cortex and in order to keep the dataset rather compact, we focused on neuronal models in layer 5 (L5). There are 13 types of morphologies (m-type) in L5: 9 types are inhibitory neurons (BP, BTC, ChC, DBC, LBC, MC, NBC, NGC, SBC) and 4 types are excitatory, i.e. the pyramidal cells, (STPC, TTPC1, TTPC2, UTPC). Since the aim of this work is to estimate the soma location, the prediction algorithm does not differentiate among morphologies, but it finds a 'universal' model to estimate the neuron position. Although inhibitory neurons having the same morphology can have different electrophysiology properties (e-type - e.g. bAC: burst accomodating) based on their firing patterns [7], we used a single e-type for each morphology, since a single spike waveform is used to triangulate the neuron location. The 13 neuron model names, available online at [8], are shown in Table I.

\section{B. Simulated Recordings}

The cell models, including morphological and biophysical information, are compiled using NEURON [9] and simulated using LFPy [10]. Each of the 13 neurons is simulated

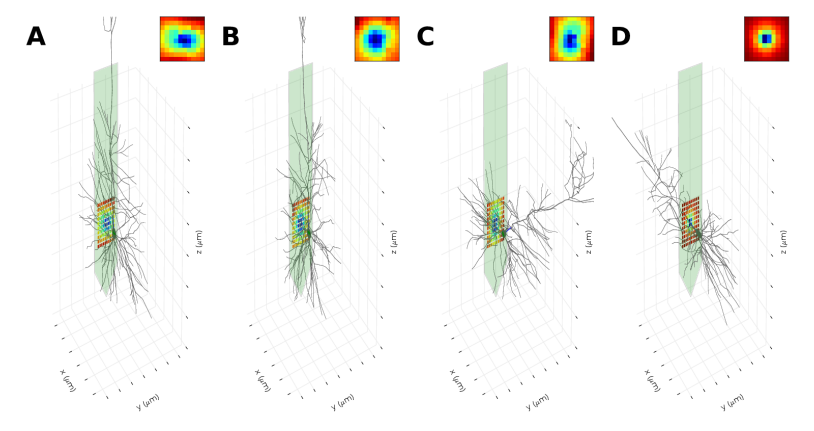

Fig. 1. 3D representation of the possible relative alignments of a pyramidal cell (TTPC1) with soma at $(50 \mu \mathrm{m}, 0,0)$ with respect to the MEA in the Norot (A), Zrot (B), Xrot (C), and 3drot (D) datasets. The psudocolors of the recording sites and the upper right axes are the sodium trough images. The images of Norot, Zrot, and Xrot datasets have quite similar shapes and extensions, while the in the 3 drot case the peak is more circumscribed mainly due to the different inclination of the soma. separately: a constant depolarizing current is applied to the soma so that the neuron fires 10 times in the simulation period $(1.2 \mathrm{~s})$; spikes are then clipped between $\mathrm{t}=-2 \mathrm{~ms}$ and $\mathrm{t}=5 \mathrm{~ms}$ where $\mathrm{t}=0$ is the time of the intracellular membrane voltage peak; for each spike the transmembrane currents of all compartments as well as the somatic membrane potential are saved. Transmembrane currents are used within LFPy to build the extracellular potential at the recording sites by linearly summing the contribution of each segment using a line source model (tissue conductivity $\sigma=0.3 \mathrm{~S} / \mathrm{m}$ - for further detail please refer to [5], [10]).

As mentioned above, the MEA follows the prototype described in [1]: recording sites are in a 2D configuration with an inter-electrode-distance of $15 \mu \mathrm{m}$, but instead of a $16 \times 16$ configuration, we used a $10 \times 10$ configuration. The reference system is chosen so that the center of the MEA is located at the origin, the recording sites are on the $y z$-plane and neurons are located in the semispace having positive $x$ axis (the $x$-coordinate is thus the distance from the MEA). For each neuron, 1000 extracellular action potential (AP) recordings are simulated by randomly choosing 1 out of the 10 generated spikes and by placing the soma at random 3D locations within the following boundaries: $x \in[10 \mu \mathrm{m}, 60 \mu \mathrm{m}]$, $y \in[-75 \mu \mathrm{m}, 75 \mu \mathrm{m}]$, and $z \in[-75 \mu \mathrm{m}, 75 \mu \mathrm{m}]$ (the MEA covers the $y z$ plane from around $-75 \mu \mathrm{m}$ to $75 \mu \mathrm{m}$ in the $y$ and $z$ directions). When computing the extracellular potential, LFPy automatically adjusts the position of neuron segments overlapping a recording point, which would lead to an infinite potential. Moreover, only locations and spikes generating a perceptible spike waveform, with a peak-to-peak amplitude of $30 \mu \mathrm{V}$ or more, are included in the dataset. As anticipated in section I, we investigate different neuron-MEA alignments or rotations. Some neurons, such as pyramidal or bipolar cells, have morphologies that follow a specific orientation that might affect the localization performance, since all neuron compartments contribute to the extracellular potential [6]. For this reason, we generated four datasets: 1) Norot: neurons are only moved around the MEA and the orientation of PC apical dendrite is along the $z$-axis; 2) Zrot: neurons are moved around the MEA and rotated randomly around the $z$-axis 3) Xrot: neurons are moved around the MEA and rotated randomly around the $x$-axis 4) 3drot: neurons are moved around the MEA and rotated randomly around all axes. Fig. 1 shows how a pyramidal cell (TTPC1) can be oriented with respect to the MEA in each of the four datasets (A - Norot, B - Zrot, C - Xrot, D - 3drot).

With the simulated signals, generated from forward modeling of the neuron's electrophysiology as described above, it is time to extract significant features and estimate the somata locations.

\section{Deep Learning Approach}

As introduced in section $\mathrm{I}$, in order to add value to the rich spatial information conveyed by the high density MEA, a Convolutional Neural Network (CNN) has been chosen as machine learning algorithm. CNNs are used because they should be shift and space invariant, due to the convolution 
operations and the local connectivity of the convolutional layers.

As input for the network we used two different sets of features: in the first one, a single time point corresponding to the minimum potential on the MEA signals is used, which should correspond to the sodium trough $(\mathrm{Na})$; in the second, we extracted both the sodium trough image and the repolarisation peak (NaRep). Since the vicinity of the dendritic tree to the MEA might cause an initial extracellular positive peak, followed by the negative peak due to outward capacitive currents in the passive membrane segments (balancing the large sodium inward current in the soma), the time point of the repolarisation peak is selected when the maximum potential after the sodium trough has occured. These time points are chosen because during these phases of the AP the larger currents are confined at the soma.

Tensorflow [11] has been used to train the CNN, which has the following topology. The 10x10 voltage image(s) (see Fig. 1) go into a 32-deep convolutional ReLU layer which filters the input image with $3 \times 3$ kernels with stride $=1$; then max pooling is applied and the image is shrinked to a $5 \times 5$; another 64-deep convolutional ReLU layer applies 3x3 kernels; max pooling reduces the output image features to a $3 \times 3$ size. The $3 \times 3$ features are input for a fully connected layer with 1024 artificial neurons and 3 output nodes linearly sum their inputs and biases to output the $x$-, $y$-, and $z$-coordinates. In order to avoid overfitting, the dropout method is implemented with a dropout rate of 0.7 . The training is run with the Adam optimizer for 5000 epochs, each time sampling 1000 observations from the dataset.

The model is validated by leaving out $10 \%$ of the observations (1300 out of 13000) and then by computing the average total error and standard deviation of the error distribution. Errors along the different axes are also computed, and the error trend with respect to the distance from the MEA and the neuron morphology is analyzed in the next section.

\section{RESUlTS}

\section{A. Localization with different rotations}

The first question is to investigate whether the alignment between neurons and the MEA is important for prediction performance. By looking at Fig. 1, we could expect that Norot would yield the best results, because neurons are only shifted and not rotated. The Zrot and Xrot datasets should also be better than 3 drot one, because neurons are only rotated with respect to one axis only. In Table II the prediction performance expressed in average error \pm error standard deviation is shown for the total error and the errors along the three axes. Moreover, the performance using the two feature sets, $\mathrm{Na}$ or NaRep, as explained in section II-C, is shown.

As expected, the errors are lower for the Norot dataset, while they increase for Zrot, Xrot and especially for 3 drot datasets. For all four datasets, the performance of NaRep feature set, i.e. using both the sodium trough and the repolarisation peak time points, is better than using the sodium peak alone. The total error, which is the actual distance between

\begin{tabular}{cccccc} 
dataset & features & error $(\mu \mathrm{m})$ & $\boldsymbol{x}$ error $(\mu \mathrm{m})$ & $\boldsymbol{y}$ error $(\mu \mathrm{m})$ & $z$ error $(\mu \mathrm{m})$ \\
\hline Norot & $\mathrm{Na}$ & $3.03 \pm 2.88$ & $1.54 \pm 2.2$ & $1.18 \pm 1.22$ & $1.78 \pm 2.05$ \\
Zrot & $\mathrm{Na}$ & $5.2 \pm 5.57$ & $2.36 \pm 3.61$ & $2.5 \pm 3.88$ & $2.82 \pm 3.18$ \\
Xrot & $\mathrm{Na}$ & $9.17 \pm 6.09$ & $3.09 \pm 3.36$ & $5.12 \pm 4.77$ & $5.31 \pm 4.81$ \\
3drot & $\mathrm{Na}$ & $11.13 \pm 8.99$ & $5.98 \pm 6.05$ & $5.5 \pm 6.06$ & $5.12 \pm 6.26$ \\
Norot & NaRep & $2.51 \pm 2.0$ & $1.09 \pm 1.18$ & $1.13 \pm 1.19$ & $1.53 \pm 1.64$ \\
Zrot & NaRep & $4.3 \pm 4.03$ & $1.99 \pm 2.26$ & $2.23 \pm 2.63$ & $2.18 \pm 3.0$ \\
Xrot & NaRep & $6.6 \pm 4.31$ & $2.24 \pm 2.32$ & $3.75 \pm 3.25$ & $3.71 \pm 3.65$ \\
3drot & NaRep & $8.21 \pm 6.68$ & $4.24 \pm 4.54$ & $4.09 \pm 4.26$ & $3.98 \pm 4.78$
\end{tabular}

TABLE II

PREDICTION PERFORMANCE ON DIFFERENT DATASETS WITH DIFFERENT FEATURE SETS IN TERMS OF TOTAL ERROR (ERROR) AND ERRORS ON DIFFERENT AXES. THE VALUES REPRESENT THE AVERAGE \pm STANDARD DEVIATION OF THE ERROR DISTRIBUTION ON THE VALIDATION SET.

the true position and the estimated position, for the Norot dataset is very low, down to $2.51 \pm 2.0 \mu \mathrm{m}$. Following is the Zrot dataset, with an error of $4.3 \pm 4.03 \mu \mathrm{m}$. It should be noticed that in most of the cases the insertion angle of the MEA and the relative alignment with the tissue is known, hence the Zrot situation might be not too far from a real scenario.

\section{B. Error versus distance from MEA}

Fig. 2A shows the distribution of the total error using the NaRep feature set with respect to the $x$-coordinate. The $x$-axis from 10 to $60 \mu \mathrm{m}$ has been binned into 5 bins of $10 \mu \mathrm{m}$ width and the mean and standard deviation of the errors have been computed for each bin (the mean is the marker, the standard deviation is the error bar). It can be seen that the prediction tends to degrade (increased average error and standard deviation) with increasing distance from the MEA, as observed in [5]. When looking at the distribution of the predicted depth with respect to the true $x$-coordinate in Fig. 2B, one can notice how the $\mathrm{x}$ error is mainly due to an underestimate of the $x$ position with increasing distance.
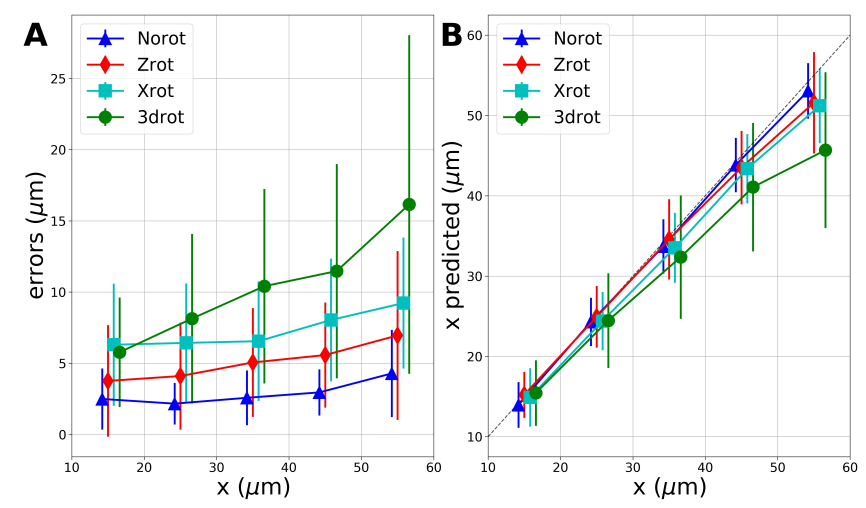

Fig. 2. (A) Trend of the error distribution (using NaRep feature set) with respect to distance from the MEA $(x)$. Markers are the average of the error distribution and errorbars represent the standard deviations. The blue, red, cyan, and green lines show the Norot, Zrot, Xrot, and 3drot datasets, respectively. (B) Predicted distance ( $x$ predicted) in function of true distance $(x)$ with same notation as in A. The errors tend to increase with increasing distance to the MEA (A), mainly due to an underestimation at large distances (B). 


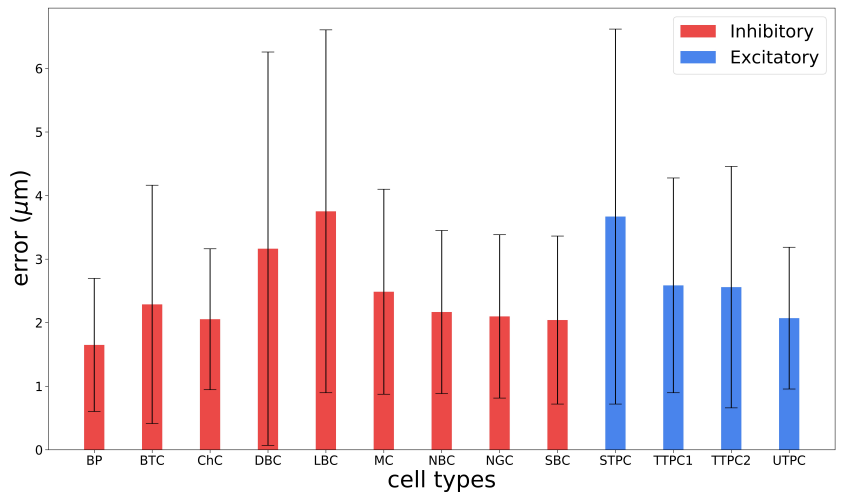

Fig. 3. Barplot showing the error average and standard deviation (errorbar) of the Norot dataset using NaRep features depending on the cell morphology. All error averages except LBC and STPC are between 1.65 and $3.5 \mu \mathrm{m}$ showing a consistent performance among cell types. Red bars show inhibitory neurons, while blue bars represent excitatory ones.

\section{Error versus cell type}

In Fig. 3 the barplot shows the average total error and its standard deviation divided by neuron morphology type (13 morphologies as explained in section II-A) for the Norot dataset using NaRep feature set. The minimum error of $1.65 \pm 1.05 \mu \mathrm{m}$ is obtained for the BP morphology, while the worse performance is $3.75 \pm 2.85 \mu \mathrm{m}$ for LBC morphology. The difference in prediction performance with respect to cell type does not seem to be depending on excitatory/inhibitory morphologies (i.e. pyramidal and non-pyramidal cells) nor do they look to be clustered depending on morphological sub-classes, for instance in basket cells (BC) there is some variability among $\mathrm{LBC}, \mathrm{NBC}$ and $\mathrm{SBC}$ and the same holds for pyramidal cells. Further analysis in this direction could give interesting insights on the precise role of neuron morphologies on prediciton performance. Differently than in [5], in which there was a wide variability in performance depending on neuron morphologies, here the models are less morphology-sensitive and more consistent among cell types.

\section{DISCUSSIONS AND CONCLUSION}

The proposed approach combines detailed biophysical forward modeling and powerful machine learning techniques to estimate 3D somata locations from MEA simulated recordings. The results show that the prediction error is very low with respect to other state-of-the-art methods and it depends on the relative alignment between neurons and MEA.

The findings, though, are only based on simulations, but here high-detailed models are used instead of simpler ones [4], [5]. A straightforward improvement to obtain more accurate simulations could be including the MEA scar in the data generation, by clipping or bending neuronal branches in the proximity of the probe before simulating the recordings. In this work we did not include any noise in the simulated recordings, with the rationale that sorted spikes can be cleaned by applying spike-triggered-averaging. Of course, validation on real data is required and we are working on paired approaches with either electrophysiological recordings only [12], or involving also calcium/voltage imaging.
The forward modeling/machine learning paradigm could be extended to extract other information from the recordings. We are currently working on morphology classification including spike features such as amplitude and width. Moreover, simulation give important insight in order to drive the design of new probes, for example in terms of number, density, size, and location of recording sites. Another advantage of using forward modeling is that the performance of the machine learning algorithm could be improved by building case-specific datasets that better match the real recordings, depending on animal type, target layer(s), insertion angle, MEA characteristics and so on.

We envision that high-density next-generation MEAs will allow to image the neural tissue by recording, localizing, and classifying neurons; the extracted information could be used to optimize stimulation paradigms [13] in precise and highly controlled closed-loop experiments, allowing a device/tissue interaction down to the single neuron resolution.

\section{ACKNOWLEDGMENTS}

Alessio P. Buccino is a doctoral fellow in the SimulaUCSD-University of Oslo Research and PhD training (SUURPh) program, an international collaboration in computational biology and medicine funded by the Norwegian Ministry of Education and Research.

\section{REFERENCES}

[1] S. Schröder, C. Cecchetto, S. Keil, et al., "CMOS-compatible purely capacitive interfaces for high-density in-vivo recording from neural tissue," in Biomedical Circuits and Systems Conference (BioCAS), 2015 IEEE, pp. 1-4, 2015.

[2] S. Vassanelli, "Multielectrode and multitransistor arrays for in vivo recording," in Nanotechnology and Neuroscience: Nano-electronic, Photonic and Mechanical Neuronal Interfacing, pp. 239-267, Springer, 2014.

[3] T. J. Blanche, M. A. Spacek, J. F. Hetke, et al., "Polytrodes: highdensity silicon electrode arrays for large-scale multiunit recording," Journal of neurophysiology, vol. 93, no. 5, pp. 2987-3000, 2005.

[4] Z. Somogyvári, D. Cserpán, I. Ulbert, et al., "Localization of singlecell current sources based on extracellular potential patterns: the spike CSD method," European Journal of Neuroscience, vol. 36, no. 10, pp. 3299-3313, 2012.

[5] I. D. Ruz and S. R. Schultz, "Localising and classifying neurons from high density MEA recordings," Journal of Neuroscience Methods, vol. 233, pp. 115-128, 2014.

[6] K. H. Pettersen and G. T. Einevoll, "Amplitude variability and extracellular low-pass filtering of neuronal spikes," Biophysical Journal, vol. 94, no. 3, pp. 784-802, 2008.

[7] H. Markram, E. Muller, S. Ramaswamy, et al., "Reconstruction and simulation of neocortical microcircuitry," Cell, vol. 163, no. 2, pp. 456492, 2015.

[8] "https://bbp.epfl.ch/nmc-portal/welcome."

[9] N. T. Carnevale and M. L. Hines, The NEURON book. Cambridge University Press, 2006.

[10] H. Lindén, E. Hagen, S. Leski, et al., "LFPy: a tool for biophysical simulation of extracellular potentials generated by detailed model neurons," Frontiers in Neuroinformatics, vol. 7, p. 41, 2014.

[11] M. Abadi, A. Agarwal, P. Barham, et al., "TensorFlow: Large-scale machine learning on heterogeneous systems," 2015. Software available from tensorflow.org

[12] J. P. Neto, G. Lopes, J. Frazão, et al., "Validating silicon polytrodes with paired juxtacellular recordings: method and dataset," Journal of Neurophysiology, vol. 116, no. 2, pp. 892-903, 2016.

[13] A. P. Buccino, T. Stöber, S. Næss, et al., "Extracellular single neuron stimulation with high-density multi-electrode array," in 2016 IEEE Biomedical Circuits and Systems Conference (BioCAS), pp. 520-523, Oct 2016. 